\title{
Public Opinion on Nutrition-Related Policies to Combat Child Obesity, Los Angeles County, 2011
}

Paul A. Simon, MD, MPH; Choiyuk Chiang, MPH; Amy S. Lightstone, MPH; Margaret Shih, MD, $\mathrm{PhD}$

Suggested citation for this article: Simon PA, Chiang C, Lightstone AS, Shih M. Public Opinion on Nutrition-Related Policies to Combat Child Obesity, Los Angeles County, 2011. Prev Chronic Dis 2014;11:140005. DOI: http://dx.doi.org/10.5888/pcd11.140005 国.

PEER REVIEWED

\section{Abstract}

We assessed public opinion on nutrition-related policies to address child obesity: a soda tax, restrictions on advertising unhealthy foods and beverages to children, and restrictions on siting fast food restaurants and convenience stores near schools. We analyzed data from 998 adults (aged $\geq 18$ years) in the 2011 Los Angeles County Health Survey. Support was highest for advertising restrictions (74\%), intermediate for a soda tax (60\%), and lowest for siting restrictions on fast food restaurants and convenience stores ( $44 \%$ and $37 \%$, respectively). Support for food and beverage advertising restrictions and soda taxation is promising for future policy efforts to address child obesity.

\section{Objective}

Efforts to reverse the obesity epidemic are unlikely to be successful without policy interventions that address "toxic" food environments (1). Although most nutrition policies to address the child obesity epidemic have focused on school, preschool, and childcare settings, national guidelines also include policy strategies in community settings (2). Only limited information is available on the comparative levels of support among these policy options (3). To address this limitation, we examined public opinion in Los Angeles County on 4 prominent nutrition-related policy strategies in community settings to address child obesity: sugar-sweetened beverage taxation, restrictions on unhealthy food and beverage marketing to children, restrictions on fast food restaurants near schools, and restrictions on convenience stores near schools.

\section{Methods}

We analyzed data from the 2011 Los Angeles County Health Survey (LACHS), a random-digit-dialed telephone (landline and cellular) survey of the noninstitutionalized county population (4). The survey was approved by the Los Angeles County Department of Public Health's Institutional Review Board.

Among all eligible households contacted for the survey, interviews were completed with $66 \%(n=8,036)$. The present study included a randomly selected subsample of 998 adults (aged $\geq 18$ years) who were asked whether they agreed or disagreed with the following 4 statements: 1) I would support a tax increase on sodas as a way to discourage kids and others from drinking too many of them; 2) There should be restrictions placed on the advertising of sugared cereals, candy, soda, and fast food to children; 3) There should be a law prohibiting fast food restaurants within a quarter-mile of schools; and 4) There should be a law prohibiting convenience stores within a quarter-mile of schools. Interviews were conducted in English, Spanish, Cantonese, Mandarin, Vietnamese, and Korean.

The data were weighted to reduce potential bias associated with differential selection and response (4). The percentage of adults who agreed with each policy statement was calculated for the total study group and by sex, age group, race/ethnicity, education level, and annual household income relative to the federal poverty level. Ninety-five percent confidence intervals were calculated for each point estimate. All analyses were conducted by using SAS (version 9.2, SAS Institute Inc, Cary, North Carolina). 


\section{Results}

Overall, restrictions on unhealthy food and beverage advertising received the highest level of support (74\%), followed by a soda tax (60\%) (Table). Restrictions on siting fast food restaurants and convenience stores near schools had considerably less support (44\% and 37\%, respectively). Women were more supportive of the policies than men. Among Latinos, support was greater among those from Spanish-speaking households than English-speaking households for all 4 policy statements.

Variation in support was also observed across other demographic subgroups but differed by policy statement. For the soda tax, support was greater among Latinos and Asians/Pacific Islanders than among whites and African Americans and was higher among those with less education and lower household incomes. For advertising restrictions, support was high across all demographic subgroups. For restrictions on fast food restaurants and convenience stores near schools, support was lowest among whites and those with higher household incomes. Support for restrictions on convenience stores was lower among adults aged 18 to 29 years than among older adults.

\section{Discussion}

Our findings suggest that efforts to restrict unhealthy food and beverage marketing to children may have broad public support if focused specifically on advertising restrictions. There is strong evidence that this marketing has a significant adverse impact on dietary patterns in children and may be an important contributor to the obesity epidemic (5). Although there are legal considerations in pursuing public policy that would regulate food and beverage marketing to children (6), our results suggest that such efforts would receive support. This support may help persuade private companies to self-regulate (7). There may also be opportunities to expand advertising restrictions in public schools and publicly regulated child care settings. For example, the White House and US Department of Agriculture recently announced proposed federal restrictions on unhealthy food and beverage advertising in public schools (8).

We also found that nearly two-thirds of adults in the county support taxation of soda as a means of reducing its consumption. However, the defeats by wide margins of 2 sugar-sweetened beverage tax initiatives in California in 2012 (9), including 1 in the City of El Monte in Los Angeles County, suggest that this support may not be sustained in the face of strong opposition, highlighting the need for robust and well-coordinated advocacy efforts. In addition, our results suggest that these efforts are unlikely to be successful unless they are able to generate greater support among whites, African Americans, and those in middle-income and more affluent households.

The low public support for restrictions on siting fast food restaurants and convenience stores near schools suggests that using zoning and other land use regulation to improve food environments may be challenging. Because this strategy is focused primarily on new establishments, its impact may be relatively limited in urban centers where large numbers of pre-existing fast food restaurants and convenience stores are near schools.

Our study has several limitations. First, although Los Angeles County comprises an ethnically and socioeconomically diverse urban, suburban, and rural population, the results may not be generalizable to other jurisdictions. For example, a national online survey of parents found only modest support for food advertising restrictions, although the measures used were different from our survey and the results, therefore, are not directly comparable (10). In addition, our findings on food advertising restrictions and sugar-sweetened beverage taxation are consistent with those of several statewide surveys in California $(11,12)$, suggesting that our results may reflect more widespread views. Second, because the survey collected only information on whether respondents agreed or disagreed with the policy statements, we cannot assess the intensity of support or opposition to these statements. Despite this limitation, our results indicate the levels of support across the 4 policy areas, providing direction for prioritizing policy efforts.

\section{Acknowledgments}

The authors did not receive grant or other external funding support for this work.

\section{Author Information}

Corresponding Author: Paul A. Simon, MD, MPH, Director, Division of Chronic Disease and Injury Prevention, Los Angeles County Department of Public Health, 3530 Wilshire Blvd, 8th Floor, Los Angeles, CA 90010. Telephone: 213351-7825. E-mail: psimon@ph.lacounty.gov.

Author Affiliations: Choiyuk Chiang, Amy S. Lightstone, Margaret Shih, Los Angeles County Department of Public Health, Los Angeles, California.

\section{References}


1. Story M, Kaphingst KM, Robinson-O'Brien R, Glanz K. Creating healthy food and eating environments: policy and environmental approaches. Annu Rev Public Health 2008;29:253-72. CrossRef 娄 PubMed 国

2. Institute of Medicine. Local government actions to prevent childhood obesity. Washington (DC): The National Academies Press; 2009.

3. Morain S, Mello MM. Survey finds public support for legal interventions directed at health behavior to fight noncommunicable disease. Health Aff (Millwood) 2013;32(3):486-96. CrossRef 圈 PubMed 圈

4. 2011 Los Angeles County Health Survey: survey methodology report.

http://publichealth.lacounty.gov/ha/docs/2011LACHS/LACHS\%20Methodological\%20Report_REV121029.pdf. Accessed January 3, 2014.

5. Cairns G, Angus K, Hastings G, Caraher M. Systemic reviews of the evidence on the nature, extent and effects of food marketing to children. A retrospective review. Appetite 2013;62:209-15. CrossRef 国 PubMed 圈

6. ChangeLab Solutions. First Amendment FAQs.

http://changelabsolutions.org/sites/default/files/FirstAmendmentFAQs_FINAL_201204.pdf. Accessed January 3, 2014.

7. Barnes B. Promoting nutrition, Disney to restrict junk-food ads. New York Times. June 5, 2012. http://www.nytimes.com/2012/06/05/business/media/in-nutrition-initiative-disney-to-restrictadvertising.html?pagewanted=all\&_r=0. Accessed February 28, 2014.

8. US Department of Agriculture. The White House and USDA announce school wellness standards. http://www.usda.gov/wps/portal/usda/usdahome?contentid=2014/02/o029.xml. Accessed February 28, 2014.

9. Los Angeles Times. Soda taxes lose big in California. http://latimesblogs.latimes.com/lanow/2012/11/soda-taxeslose-big-in-california.html. Accessed January 3, 2014.

10. Goren A, Harris JL, Schwartz MB, Brownell KD. Predicting support for restricting food marketing to youth.

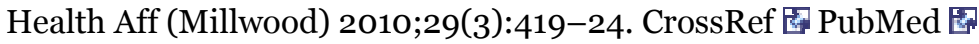

11. The Field Poll. Voter concerns about risk factors for obesity and diabetes have eclipsed other health concerns facing California kids over the past 10 years. http://www.field.com/fieldpollonline/subscribers/Rls246o.pdf. Accessed February 28, 2014.

12. The Field Poll. Most Californians see a direct linkage between obesity and sugary sodas. Two in three voters support taxing sugar-sweetened beverages if proceeds are tied to improving school nutrition and physical activity programs. http://field.com/fieldpollonline/subscribers/Rls2436.pdf. Accessed February 28, 2014.

\section{Table}

Table. Respondents Who Agreed with Nutrition Policy Statements by Sociodemographic Characteristic, Los Angeles County Health Survey, 2011

\begin{tabular}{|c|c|c|c|c|c|c|c|c|}
\hline \multirow[b]{2}{*}{ Characteristic } & \multicolumn{2}{|c|}{ Soda Tax } & \multicolumn{2}{|c|}{$\begin{array}{l}\text { Restrict Food } \\
\text { Marketing }\end{array}$} & \multicolumn{2}{|c|}{$\begin{array}{l}\text { Prohibit Fast Food } \\
\text { Near Schools }\end{array}$} & \multicolumn{2}{|c|}{$\begin{array}{l}\text { Prohibit Convenience } \\
\text { Stores Near Schools }\end{array}$} \\
\hline & na & $\begin{array}{c}\% \text { Agree } \\
\text { (95\% CI) }\end{array}$ & na & $\begin{array}{c}\% \text { Agree } \\
(95 \% \text { CI) }\end{array}$ & na & $\begin{array}{c}\% \text { Agree } \\
(95 \% \text { CI) }\end{array}$ & na & $\%$ Agree $(95 \% \mathrm{CI})$ \\
\hline Overall & 972 & $\begin{array}{r}60.1(55.3- \\
64.8)\end{array}$ & 973 & $\begin{array}{r}74.2(70.0- \\
78.4)\end{array}$ & 957 & $\begin{array}{r}43.5(38.5- \\
48.5)\end{array}$ & 956 & $37.0(32.2-41.9)$ \\
\hline \multicolumn{9}{|l|}{ Sex } \\
\hline Male & 371 & $\begin{array}{r}57.0(49.5- \\
64.6)\end{array}$ & 370 & $\begin{array}{r}68.2(61.0- \\
75.3)\end{array}$ & 366 & $\begin{array}{r}37.2(29.7- \\
44.8)\end{array}$ & 368 & $34.8(27.3-42.3)$ \\
\hline Female & 601 & $\begin{array}{r}62.9(57.1- \\
68.8)\end{array}$ & 603 & $\begin{array}{r}79.7(75.1- \\
84.4)\end{array}$ & 591 & $\begin{array}{r}49.3(42.8- \\
55.7)\end{array}$ & 588 & $39.2(32.8-45.5)$ \\
\hline \multicolumn{9}{|l|}{ Age group, y } \\
\hline $18-29$ & 121 & $\begin{array}{r}61.1(49.6- \\
72.6) \\
\end{array}$ & 123 & $\begin{array}{r}69.5(58.3- \\
80.8)\end{array}$ & 121 & $\begin{array}{r}45.4(33.4- \\
57.5) \\
\end{array}$ & 123 & $24.5(14.9-34.1)$ \\
\hline $30-49$ & 358 & $\begin{array}{r}63.4(55.9- \\
70.9)\end{array}$ & 358 & $\begin{array}{r}78.5(72.7- \\
84.3)\end{array}$ & 355 & $\begin{array}{r}43.6(35.4- \\
51.7)\end{array}$ & 356 & $42.2(33.9-50.5)$ \\
\hline $50-64$ & 268 & & 267 & & 265 & & 261 & $39.4(30.7-48.1)$ \\
\hline
\end{tabular}


Preventing Chronic Disease | Public Opinion on Nutrition-Related Policies to Combat Chi... Page 4 of 5

\begin{tabular}{|c|c|c|c|c|c|c|c|c|}
\hline \multirow[b]{2}{*}{ Characteristic } & \multicolumn{2}{|c|}{ Soda Tax } & \multicolumn{2}{|c|}{$\begin{array}{l}\text { Restrict Food } \\
\text { Marketing }\end{array}$} & \multicolumn{2}{|c|}{$\begin{array}{l}\text { Prohibit Fast Food } \\
\text { Near Schools }\end{array}$} & \multicolumn{2}{|c|}{$\begin{array}{l}\text { Prohibit Convenience } \\
\text { Stores Near Schools }\end{array}$} \\
\hline & na & $\begin{array}{l}\text { \% Agree } \\
\text { ( } 95 \% \text { CI })\end{array}$ & na & $\begin{array}{l}\text { \% Agree } \\
\text { (95\% CI) }\end{array}$ & na & $\begin{array}{l}\text { \% Agree } \\
\text { (95\% CI) }\end{array}$ & na & $\%$ Agree $(95 \% \mathrm{CI})$ \\
\hline & & $\begin{array}{r}57.1(48.6- \\
65.7)\end{array}$ & & $\begin{array}{r}70.7(62.9- \\
78.5)\end{array}$ & & $\begin{array}{r}42.3(33.4- \\
51.2)\end{array}$ & & \\
\hline$\geq 65$ & 225 & $\begin{array}{r}53.4(43.2- \\
63.6)\end{array}$ & 225 & $\begin{array}{r}75.8(67.1- \\
84.5)\end{array}$ & 216 & $\begin{array}{r}42.0(31.8- \\
52.2)\end{array}$ & 216 & $40.8(30.6-51.1)$ \\
\hline \multicolumn{9}{|l|}{ Race/Ethnicity } \\
\hline Latino & 327 & $\begin{array}{r}68.1(60.6- \\
75.6)\end{array}$ & 325 & $\begin{array}{r}76.0(69.2- \\
82.8) \\
\end{array}$ & 325 & $\begin{array}{r}53.5(45.1- \\
61.8)\end{array}$ & 319 & $38.3(30.5-46.0)$ \\
\hline White & 431 & $\begin{array}{r}46.5(39.0- \\
54.0)\end{array}$ & 429 & $\begin{array}{r}74.6(68.9- \\
80.3)\end{array}$ & 424 & $\begin{array}{r}30.9(24.3- \\
37.4)\end{array}$ & 427 & $26.8(19.6-34.1)$ \\
\hline African American & 113 & $\begin{array}{r}50.2(36.8- \\
63.5)\end{array}$ & 113 & $\begin{array}{r}72.3(59.5- \\
85.1)\end{array}$ & 109 & $\begin{array}{r}44.7(31.2- \\
58.1)\end{array}$ & 108 & $41.1(27.7-54.5)$ \\
\hline Asian/Pacific Islander & 91 & $\begin{array}{r}71.6(59.7- \\
83.4)\end{array}$ & 96 & $\begin{array}{r}70.2(56.8- \\
83.5)\end{array}$ & 89 & $\begin{array}{r}41.3(27.2- \\
55.5)\end{array}$ & 92 & $53.7(39.4-68.1)$ \\
\hline \multicolumn{9}{|c|}{ Language of interview among Latinos } \\
\hline English-speaking & 178 & $\begin{array}{r}52.9(41.0- \\
64.8)\end{array}$ & 178 & $\begin{array}{r}67.6(56.5- \\
78.7)\end{array}$ & 177 & $\begin{array}{r}40.8(29.5- \\
52.2)\end{array}$ & 179 & $28.1(18.9-37.2)$ \\
\hline Spanish-speaking & 149 & $\begin{array}{r}79.5(70.6- \\
88.4)\end{array}$ & 147 & $\begin{array}{r}82.4(74.2- \\
90.7)\end{array}$ & 148 & $\begin{array}{r}62.8(51.7- \\
73.9)\end{array}$ & 140 & $46.5(35.0-58.0)$ \\
\hline \multicolumn{9}{|l|}{ Education } \\
\hline $\begin{array}{l}\text { Less than high school } \\
\text { graduate }\end{array}$ & 163 & $\begin{array}{r}72.6(63.2- \\
82.0)\end{array}$ & 164 & $\begin{array}{r}81.7(73.5- \\
89.9)\end{array}$ & 161 & $\begin{array}{r}56.0(446- \\
67.5)\end{array}$ & 157 & $39.9(28.8-50.9)$ \\
\hline High school graduate & 151 & $\begin{array}{r}66.5(56.2- \\
76.8)\end{array}$ & 152 & $\begin{array}{r}70.5(59.7- \\
81.3)\end{array}$ & 144 & $\begin{array}{r}49.7(37.5- \\
61.9)\end{array}$ & 147 & $42.9(31.0-54.9)$ \\
\hline $\begin{array}{l}\text { Some college or trade } \\
\text { school }\end{array}$ & 272 & $\begin{array}{r}46.8(38.3- \\
55.3)\end{array}$ & 272 & $\begin{array}{r}71.8(63.9- \\
79.6)\end{array}$ & 268 & $\begin{array}{r}40.2(31.7- \\
48.7)\end{array}$ & 269 & $33.2(25.2-41.2)$ \\
\hline $\begin{array}{l}\text { College or } \\
\text { postgraduate degree }\end{array}$ & 379 & $\begin{array}{r}58.1(49.8- \\
66.4)\end{array}$ & 379 & $\begin{array}{r}73.1(66.1- \\
80.1)\end{array}$ & 377 & $\begin{array}{r}30.7(23.4- \\
38.1)\end{array}$ & 377 & $33.7(24.9-42.6)$ \\
\hline \multicolumn{9}{|l|}{ Incomeb } \\
\hline $0 \%-99 \% \mathrm{FPL}$ & 161 & $\begin{array}{r}69.2(58.7- \\
79.7)\end{array}$ & 162 & $\begin{array}{r}69.9(59.3- \\
80.4)\end{array}$ & 156 & $\begin{array}{r}58.5(46.9- \\
70.2)\end{array}$ & 157 & $45.0(33.4-56.6)$ \\
\hline $100 \%-199 \% \mathrm{FPL}$ & 193 & $\begin{array}{r}73.7(64.9- \\
82.6)\end{array}$ & 192 & $\begin{array}{r}81.9(73.5- \\
90.4)\end{array}$ & 187 & $\begin{array}{r}48.0(36.7- \\
59.3)\end{array}$ & 187 & $43.4(32.1-54.6)$ \\
\hline $200 \%-299 \%$ FPL & 126 & $\begin{array}{r}48.3(35.5- \\
61.0)\end{array}$ & 127 & $\begin{array}{r}71.6(59.2- \\
84.0) \\
\end{array}$ & 126 & $\begin{array}{r}45.9(33.1- \\
58.7)\end{array}$ & 124 & $34.6(22.3-46.9)$ \\
\hline $300 \%$ or above FPL & 492 & $\begin{array}{r}49.3(42.5- \\
56.1)\end{array}$ & 492 & $\begin{array}{r}72.0(66.4- \\
77.6)\end{array}$ & 488 & $\begin{array}{r}31.7(25.5- \\
37.9)\end{array}$ & 488 & $29.3(23.3-35.3)$ \\
\hline
\end{tabular}

Abbreviation: CI, confidence interval.

a Counts may not sum to overall totals because of missing data.

b Income was categorized based on US Census 2009 federal poverty level (FPL) thresholds, which for a family of 4 (2 adults, 2 dependents) correspond to annual incomes of $\$ 21,756$ (100\% FPL), $\$ 43,512$ (200\% FPL), and $\$ 65,268$ (300\% FPL).

The opinions expressed by authors contributing to this journal do not necessarily reflect the opinions of the U.S. Department of Health and Human Services, the Public Health Service, the Centers for Disease Control and Prevention, or the authors' affiliated institutions. 
Preventing Chronic Disease | Public Opinion on Nutrition-Related Policies to Combat Chi... Page 5 of 5

For Questions About This Article Contact pcdeditor@cdc.gov

Page last reviewed: June 05, 2014

Page last updated: June 05, 2014

Content source: National Center for Chronic Disease Prevention and Health Promotion

Centers for Disease Control and Prevention 1600 Clifton Rd. Atlanta, GA 30333, USA

800-CDC-INFO (800-232-4636) TTY: (888) 232-6348 - Contact CDC-INFO

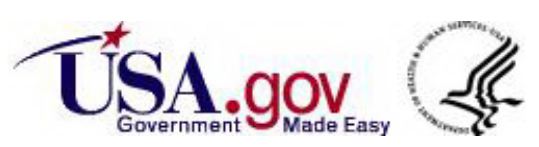

